\title{
An Occupant-participatory Approach for Thermal Comfort Enhancement and Energy Conservation in Buildings
}

\author{
Abraham Hang-yat Lam§ , Yi Yuan* and Dan Wang* \\ Department of Computing, The Hong Kong Polytechnic University* \\ The Hong Kong Polytechnic University Shenzhen Research Institute \\ Building Integration Perfection Ltd. ${ }^{\S}$ \\ MEGA Automation Ltd. \\ \{cshylam,csyiyuan,csdwang\}@comp.polyu.edu.hk
}

\begin{abstract}
Commercial building is one of the major energy consumers worldwide. Among the building services, the heating, ventilating and air-conditioning (HVAC) system dominates the total energy consumption. Recent studies have proposed various approaches to audit, automate and optimize energy usage of the HVAC system. Nevertheless, these schemes seldom discuss human thermal comfort. To minimize complaints, the current practice of the facility management is to adopt very conservative temperatures, leading to massive waste of energy.

In this paper, we actively take thermal comfort into consideration. We propose a participatory approach allowing the occupants provide feedback regarding their comfort levels. A major challenge for a participatory design is to reduce intrusiveness of the system. To this end, we develop a temperature comfort correlation model that can build a profile for each occupant. The decision of setpoint temperature can be primarily model-driven, requiring minimal inputs of the occupants. We validated our model with field experiments. Besides, we developed a setpoint optimization algorithm to handle the diverging thermal requirements of multiple occupants in same room, and examined the model with simulations. We implemented our design and conducted field experiments in a University and a commercial office. Results showed that our algorithm can successfully maintain high thermal comfort, while reducing $18 \%$ of energy consumption.
\end{abstract}

\section{Categories and Subject Descriptors}

H.1.2 [Models and Principles]: User/Machine Systems

\section{Keywords}

Participatory Thermal Comfort, Energy Conservation, Smart Building

Permission to make digital or hard copies of all or part of this work for personal or classroom use is granted without fee provided that copies are not made or distributed for profit or commercial advantage and that copies bear this notice and the full citation on the first page. Copyrights for components of this work owned by others than ACM must be honored. Abstracting with credit is permitted. To copy otherwise, or republish, to post on servers or to redistribute to lists, requires prior specific permission and/or a fee. Request permissions from permissions@acm.org.

e-Energy'14, June 11-13, 2014, Cambridge, UK.

Copyright 2014 ACM 978-1-4503-2819-7/14/06 ...\$15.00.

http://dx.doi.org/10.1145/2602044.2602067.

\section{INTRODUCTION}

In recent years, people have been paying more attentions to energy conservation around the world. The largest sectors of energy consumption are commercial buildings, residential houses, transportation, and manufactory industry. In Hong Kong, where the industry sectors are small, commercial buildings account for more than $65 \%$ of energy consumption of the city [1]. In a typical building, the HVAC (heating, ventilation, and air conditioning) system dominates the energy expenses. It is reported in the Office Segment of Hong Kong 2013 that 53\% energy have been consumed in room conditioning [1]. As a result, numbers of recent studies have proposed the idea of energy conservation by intelligently managing the HVAC systems [2][3].

Energy conservation is on one end of the spectrum. Clearly, we can simply turn-off all the air-conditioning, thus maximizing energy saving ${ }^{1}$. Nevertheless, the HVAC systems are designed to provide a comfort indoor environment for occupants in buildings. However, complaint minimization, rather than energy conservation, is the top priority of buildings and building operators. Therefore, it is important to take human thermal comfort into consideration.

The current practice of supporting human thermal comfort by building operators is to apply a fixed setpoint temperature. These temperatures are derived from large-scaled field surveys or laboratory experiments. Such recommendation provides building operators with a benchmark in temperature settings and assists them to cope with complaints. To minimize the number of complaints, these recommended temperatures are usually very conservative (i.e., the setting is on the low temperature side) and uniformly apply to the entire building unless special requests are made. However, this traditional practice has led to massive waste of energy. In addition, a lower temperature does not necessarily reflect better human thermal comfort. What is more, it is difficult for occupants to adjust the temperature on their own in many high-end buildings with the installation of the centralized HVAC system.

As opposed to such fixed setpoint strategy, there are proposals on dynamical control of the HVAC systems. One direction is to detect human presence. If a room is not occupied, the air-conditioning of the room will be turned-off.

\footnotetext{
${ }^{1}$ To ease our presentation, in this paper, we use airconditioning to represent the HVAC systems. In the context of air-conditioning, setting a lower temperature means more energy has to be consumed.
} 
Various detection objectives and solutions have been proposed in previous studies [4][5].

In this paper, we explore another direction on dynamic control of the HVAC systems. Rather than passively detecting human presence or comfort levels, we take a participatory approach in which occupants can provide inputs. More specifically, occupants can actively provide feedback on the comfort level with the use of their smartphones. The idea is simple, yet for a participatory design to succeed, we face several challenges: 1) the incentives of occupants are important. The design should be as non-intrusive as possible. Requesting occupants to provide feedback via their phones every time they stay in a room will possibly discourage participation of people. Besides, it is of great importance to protect one's privacy; 2) occupants are insensitive to the numerical expression of temperature [6]. For instance, one may not be able to differentiate the actual differences between $22.5^{\circ} \mathrm{C}$ and $24.5^{\circ} \mathrm{C}$. However, current building management system (BMS) requires numerical values for calculation and comparison. Therefore, it is necessary to have a context-aware translation; 3) it is possible to have multiple occupants in a room, thus an optimized aggregation of different comfort levels is needed; 4) it is necessary to develop a system consisting of data collection, smartphone application, interaction with building controls for air-conditioning adjustment, etc. Many of these challenges are similar to those of typical participatory sensing systems [7][8], but are specific in the building environment.

To handle these challenges, we first made use of the comfort index in linking the human thermal comfort with numerical values. We then developed a temperature-comfort correlation model to create a profile for each individual occupant. In that sense, the air-conditioning adjustment decisions are primarily model-driven, substantially reducing human feedback. Our model has referred to the thermal comfort model from the inter-discipline of built environment, where they require to collect different kinds of data with the use of special equipment. We made careful simplification on the models that the required parameters are available in a typical BMS of buildings. We developed a setpoint optimization algorithm to cater the comfort requirements for multiple occupants, and conducted a series of real-world experiments to validate our temperature-comfort correlation model. It is showed that our model has a high accuracy in predicting the occupants' thermal preference. A comprehensive set of simulations have been utilized to study thermal comfort levels and energy conservation. We implemented our design with a smartphone application, a wireless sensing system to collect necessary environmental data and a system that interacts with the BMS. We have also conducted experiments in our university and a commercial office and achieved a $18 \%$ of energy saving. The result indicates the effectiveness of our participatory approach.

\section{BACKGROUND AND RELATED WORK}

Recently, there are many studies from computer science researchers on smart buildings. The studies start from energy auditing systems using wireless sensor networks [9][10], provding fine-grained data regarding energy usage. In addition, there are studies on smart wireless systems for better automation and control of building equipment [11]. Numerous studies have also been proposed on human detection [12][5][13] of which lighting and air-conditioning can be turned-off in a smarter way. There are also studies on more intelligent arrangement of human activities such as meetings and classes with the objective in minimizing energy or electricity bills [14][4]. However, these studies have not considered human thermal comfort, which is the gap of the existing studies we seek to fill in this paper. One possible difficulty is that human thermal comfort is not immediately quantitative to computer scientists.

As a matter of fact, there are tons of studies on human thermal comfort in built environment. These studies can be summarized into two approaches [15]: the heat-balanced approach and the adaptive approach. The heat-balanced approach, first studied by Fanger in 1970 [16], observes the linkage between thermal comfort and physiological factors such as skin temperature and sweat rate. It establishes a thermal comfort model with factors such as air temperature, clothing insulation, metabolic rate, etc. To average the comfort level of all people, a predicted mean vote (PMV) model is proposed. The adaptive approach considers such factors as adaptations, e.g., behavioural and psychological adaptations [15], social and cultural background towards the thermal expectations, and also physical stimuli in response to the changing indoor and outdoor temperatures [17]. The objective of these studies is to derive a comfort temperature to the occupant, either in his activity and physiological perspective, or from his behavior, cultural background and physical environment perspective. With the objectives to improve the predictability of the models, the state-of-the-art models require many associated parameters that are complicated to obtain [18]. Besides, these experiments have heavily relied on advanced equipment for measurement. The heat-balance approach conducts experiments in laboratory with climate chamber to simulate different combination of environmental conditions, whereas adaptive approach conducts massive field studies in different regions and countries. Noted that these studies are not easy to be implemented in daily life and hence discrete from the actual operation of BMS.

As a result, there is a clear separation where the advances of smarter and more fine-grained building automation and control system seldom take human thermal comfort into consideration, and the studies of such modeling development are isolated from the control-loop of the room air-conditioning. In this paper, we jointly consider the two issues. From a high level point of view, our design considers the thermal feedback of occupants during the decision of setpoint temperature is made to the room. There are a few similar work, e.g., Thermovote [6] and SPOT+ [19]. However, there are two common problems in their studies. Firstly, they usually rely on an existing thermal comfort model from built environment (e.g., PMV). As discussed, some parameters of these models are not easy to be obtained in daily life. Therefore, they rely on prior-obtained fixed settings of these parameters, leading to error-prone results. Secondly, these studies usually require occupants to keep providing their feedback every time he stays in each room (when the occupant is not comfort). While it may improve the accuracy at that moment, it may also adversely discouraged the incentives of the participants in long run, which is a common problem encountered in participatory sensing systems [20][6]. Of course, the objectives of these studies differ from ours. In this paper, we propose a participatory approach addressing these two problems. 


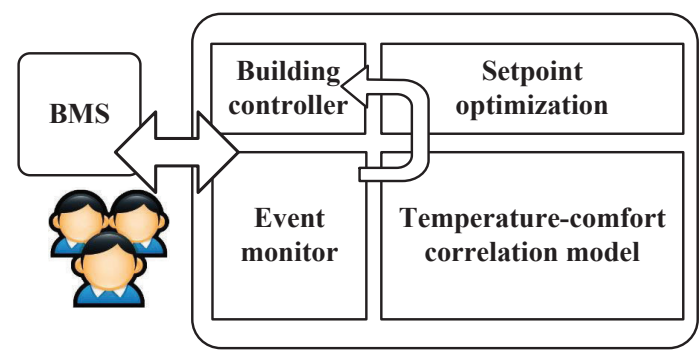

Figure 1: Overview of OPTC

\section{OCCUPANT-PARTICIPATORY THERMAL COMFORT (OPTC): AN OVERVIEW}

In this paper, we developed a smartphone application for occupants to provide their feedback (or in other words, to vote) regarding their thermal sensations to the indoor environment of buildings. The smartphone application is able to directly communicate with the BMS and thus the room setpoint temperature is adjusted accordingly. Each vote of the occupants will be recorded along with the current environmental data, e.g. indoor room temperature and outdoor temperature, to formulate that occupant's thermal comfort model. The temperature adjustment of the room can thus be model-driven most of the time with minimizing the intrusiveness to the occupant, therefore, the occupant is not expected to keep submitting their votes all the time.

In our system, we have a OPTC server to store the data collected from each occupant, including his thermal comfort profile. We first clarify the possible privacy concerns in this regard. In our design, each occupant is required to register an account with his email address and agrees a set of rules, e.g., allow us to collect his thermal comfort information. If one does not register, his thermal preference will not be taken into the consideration of our temperature adjustment. Based on our experiments, we found that all occupants have registered. Our post-experiment survey revealed that people in our experiments were not so much concerned about their thermal preferences being recorded. Instead, they were more concerned that they would be left out in our setpoint adjustment in a room of multiple people.

We have developed an Occupant-Participatory Thermal Comfort (OPTC) framework as shown in Fig. 1. There are four main modules: (1) Temperature-comfort correlation (TCC) model: the objective of TCC model is to establish a correlation (or profile) between the indoor and outdoor temperature with the comfort index of each individual occupant; (2) Setpoint optimization module: based on the TCC model, setpoint optimization computes the optimal setpoint temperature for occupant(s), especially for multiple occupants; (3) Event monitor module: the event monitor module collects data from the existing environment (e.g., indoor, outdoor temperature) and occupants information for two purposes: to trigger decision making in setpoint optimization, and to gradually train the TCC model; (4) Building controller module: After setpoint optimization module makes an adjustment decision, the building controller module communicates with the BMS to change the setpoint of the room.

Our TCC model is developed interdisciplinary with the field of built environment. However, the thermal comfort models in built environment are complicate and difficult to be implemented in real environment. Besides, the traditional thermal comfort models require tremendous field tests or laboratory experiments, which special equipment like climate chamber to simulate different climatic conditions are used. On the contrary, our model is designed to rely on daily collectable data only, e.g., indoor and outdoor temperature, and additionally occupants feedback regarding the thermal preference, though with the expectation that more feedback will be collected in the times of feeling discomfort. The feedback of each user is saved into his own profile for modeling, hence the setpoint temperature adjustment can be modeldriven most of the time. Details are shown in Section 4.

The setpoint optimization module determines the setpoint adjustment. For example, when some specified events happen (e.g., indoor/outdoor temperature change and occupants' comfort index are collected, etc.), the setpoint optimization module is then triggered. It translates the inputs from event monitor module and the TCC model into a decision for room setpoint adjustment. More importantly, we have developed an algorithm to compute the optimized setpoint temperature when there are multiple occupants sharing the same space/room. The details are demonstrated in Section 5 .

Event monitor module collects all the required data and responses accordingly. To collect the thermal preference of one, we have developed a smartphone application based on the comfort index as shown in Table 1. We have translated the fuzzy preference into computable numerical values. We elaborate the details in Section 7.

In building controller module, decisions made by the setpoint optimization module are passed to the BMS, which is discussed in Section 7.

The remaining part of the paper is organized as follows. The TCC model is discussed in Section 4, and we validated our TCC model by conducting a series of field experiments with the occupants. We then present our setpoint optimization algorithm in Section 5. In Section 6, we show a comprehensive set of simulations using our model. We have also evaluated a large sets of occupants with different physical characteristics, and evaluated the energy saving with various room configurations and sets of simulated occupants. We then present the implementation of our system in Section 7. In Section 8, we conducted two sets of experiments, one in a university and the other one in a commercial office. These experiments serve to validate our simulation results.

\section{TEMPERATURE-COMFORT CORRELA- TION (TCC) MODEL}

In essence, we need a model indicating the levels of comfort of a person, given the indoor and outdoor temperature at a specified time are known. We can then determine the setpoint temperature accordingly. As discussed before, there are many models from the discipline of built environment [21][22][23][24][16], but the accuracy of these models largely rely on real-time measurement of the occupants and the surrounding environment, where special equipment are needed.

Our choice is that we build an initial model following the rudimental laws of metabolism. Clearly, this model is still based on the discipline of built environment. In this model, we carefully categorize three different sets of parameters: 1) parameters that we can collect in one time, e.g., the age, gender, height, weight, etc of the occupant. This informa- 
Table 1: 7-point thermal comfort index

\begin{tabular}{c|c}
\hline \hline Point & Sensation \\
\hline+3 & Hot \\
+2 & Warm \\
+1 & Slightly Warm \\
0 & Neutral \\
-1 & Slightly Cool \\
-2 & Cool \\
-3 & Cold \\
\hline
\end{tabular}

tion are one-off and we collect them at the time of registration, 2) parameters that change, but can be collected by non-intrusive sensors, e.g., indoor or outdoor temperatures; 3) parameters that are hard to collect using daily sensors; and we train these parameters collectively by the votes and other parameters.

In the following parts, we first link the thermal comfort with quantifiable (numerical) values. We then develop user's thermal preference with our model. Finally we validate our model using real-world experiments.

\subsection{Thermal Comfort Metric}

To quantify thermal comfort, we adopt a seven-point thermal comfort index from the American Society of Heating, Refrigerating and Air-conditioning Engineers (ASHRAE). This index scales from -3 to 3 and links the thermal comfort from cold to hot as shown in Table 1.

From the occupants perspective, this index is easily comprehensible to reflect his thermal sensation, i.e. he can vote either hot or cold. From the built environment perspective, a value of 0 indicates that an occupant reaches thermal neutrality, meaning the heat generation and heat loss of the occupant is in the state of equilibrium. A negative index means that the occupant losses more heat than his body produces so that he feels cool or cold. The smaller the index, the more uncomfortable the occupant feels. When the index of an occupant is between -1 (Slightly Cool) and 1 (Slightly Warm), the occupant is regarded as comfort. This index range $(-1,1)$ is defined as the comfort zone [25].

\subsection{Model Development}

As explained, we first build an initial model. In this model, the thermal comfort is a function of indoor temperature $T_{i}$ of room $i$, outdoor temperature $T_{o}$ and the elapsed time $t$ a person stays at the space/room. The thermal sensation is determined by the balance of heat gain and loss of human body. Let $G(t)$ be the heat gain and $L\left(T_{i}, T_{o}\right)$ be the heat loss. Thus, the thermal comfort $C\left(T_{i}, T_{o}, t\right)$ is:

$$
C\left(T_{i}, T_{o}, t\right)=G(t)+L\left(T_{i}, T_{o}\right)
$$

In what follows, we will first present the high level ideas, followed by the details of model $G(t)$ and $L\left(T_{i}, T_{o}\right)$.

In general, the amount of heat generated is primarily settled by metabolic rate. It is also affected by the physical activity (PA) of people [26], e.g., a person has a higher metabolic rate during walking, whereas lower rate after staying sedentary. When the PA of a person changes, e.g., sit down after running, his metabolic rate changes accordingly. Thus, the occupant experiences a change of thermal sensations in the same environment. Moreover, the speed of metabolic rate change differs among individuals. In $G(t)$,

\begin{tabular}{|c|c|c|}
\hline & Men & Women \\
\hline Sedentary & 1.0 & 1.0 \\
Active & 1.25 & 1.27 \\
\hline
\end{tabular}

Table 2: Physical activity factor

we consider metabolic rate changes as elapsed time $t$ after occupant enters a room.

The amount of heat lost is determined by indoor temperature and clothes insulation. In operation, information of clothes insulation is difficult to access. However, from results of field studies [27], it is showed that the effect of clothes insulation can be reflected by outdoor temperature. Intuitively, when the outdoor temperature is higher, occupants are likely wearing less clothes, and hence prone to a higher rate of heat loss. In this regard, they prefer a warmer indoor temperature. Thus, we use indoor temperature and outdoor temperature as determining variables in $L\left(T_{i}, T_{o}\right)$.

\subsubsection{Heat Generation Modeling}

According to [28, 29], heat production is proportional to physical activity, whereas metabolic rate adjusts according to physical activity. To estimate metabolic rate of an occupant, we adopt the Estimated Energy Requirement (EER) model [30], which is first proposed by the Institute of Medicine (IOM) that used to estimate a person's daily average of dietary energy intake to maintain his energy balance. It considers the factors of gender, age, height, weight as well as the physical activity of users. EER is shown as follows:

$$
E E R=k_{1}-k_{2} \times A g e+\left[P A \times\left(k_{3} \times W+k_{4} \times H\right)\right]
$$

Here, $k_{1}$ is a constant related to gender and age. $k_{2}$ is a constant related to age. $k_{3}$ and $k_{4}$ are constants related to weight $(\mathrm{W})$ and height $(\mathrm{H})$ respectively. The $P A$ coefficient is related to the physical activity and varies with genders. As we are interested in the change of metabolic rate of a person from outdoor to indoor, we consider the coefficients of two physical activities: active and sedentary. The coefficients of active and sedentary for male and female are shown in Table 2. The details of other coefficients can be found in [30].

According to [26][25], metabolic rate changes smoothly after physical activity changes. To obtain the corresponding EER at time $t$, we formulate the EER into:

$$
E E R(t)= \begin{cases}\frac{\left(E E R_{s}-E E R_{e}\right)}{t_{c}}\left(t_{c}-t\right)+E E R_{e} & t<t_{c} \\ E E R_{e} & t \geq t_{c}\end{cases}
$$

Here, $E E R_{s}$ and $E E R_{e}$ are EER of a person at active state and sedentary state respectively. $t_{c}$ is the time required by a person to recover from active to sedentary. Beyond $t_{c}$, EER is assumed to remain as the metabolic rate becomes steady. Fig. 2 shows the EER of a male and a female with different ages, heights and weights.

Noted that $t_{c}$ is a parameter that is difficult to obtain from daily sensors. It differs from person to person as well. Intuitively, even if two people have the same weight, they still differ from muscle-fat ratio. In built environment, this is a factor of research and there are ways to estimate $t_{c}$ using laboratory equipment. As mentioned, we train $t_{c}$ using the votes from occupants. 
Table 3: Category of BMI

\begin{tabular}{|c|c|}
\hline Category & BMI Range \\
\hline Underweight & $<18.5$ \\
Normal & $18.5-25$ \\
Overweight & $>25$ \\
\hline
\end{tabular}

As described, heat production is proportional to EER. Thus we have:

$$
G(t)=a_{1} \times E \operatorname{ER}(t)+b_{1}
$$

Here, $a_{1}$ is the activity sensitivity and is associated to $t_{c}$, and $b_{1}$ is stable comfort preference. To make our presentation concise, we put the derivation of these two parameters into the Appendix.

\subsubsection{Heat Loss Modeling}

For heat loss, there are comprehensive recent findings from field studies [17][23] showing a noticeable relationship between the outdoor temperature and human thermal comfort. The correlation is formulated as $T_{c}\left(T_{o}\right)=a_{2}+b_{2} \times T_{o}$, where $T_{c}$ is the comfort temperature function of outdoor mean temperature, $a_{2}$ is a constant related to comfort temperature and $b_{2}$ is the correlation between outdoor temperature change and comfort temperature change. Intuitively, this model says that if the outdoor temperature increases, the occupants prefer higher indoor temperature. In this paper, we applied the model in [31], where $a_{2}$ is set to 17.8 and $b_{2}$ is set to 0.31 .

As specified in the standard [25], temperature range for comfort zone is $7^{\circ} \mathrm{C}$. This means that when indoor temperature increases from $T_{c}\left(T_{o}\right)-3.5^{\circ} C$ to $T_{c}\left(T_{o}\right)+3.5^{\circ} C$, the comfort index thus changes from -1 to 1 . Since $L\left(T_{i}, T_{o}\right)$ is a linear function of $T_{i}, k$ can be computed as $k=7 / 2$. Thus, $R$ can be calculated as $R=3 \times k=10.5^{\circ} C$, which is the boundaries for the comfort zone of a person. The heat loss model is thus:

$$
L\left(T_{i}, T_{o}\right)= \begin{cases}3 & T_{i}-T_{c}\left(T_{o}\right) \geq R \\ k\left(T_{i}-T_{c}\left(T_{o}\right)\right) & -R<T_{i}-T_{c}\left(T_{o}\right)<R \\ -3 & T_{i}-T_{c}\left(T_{o}\right) \leq-R\end{cases}
$$

\subsection{Model Validation}

We conducted a series of field experiments to validate our model. One experiment was conducted in a commercial office for five consecutive days, and 13 occupants were invited to participate in this experiment. We built the profile of each occupant using the TCC model and trained for three consecutive days. For the remaining two days, we compare

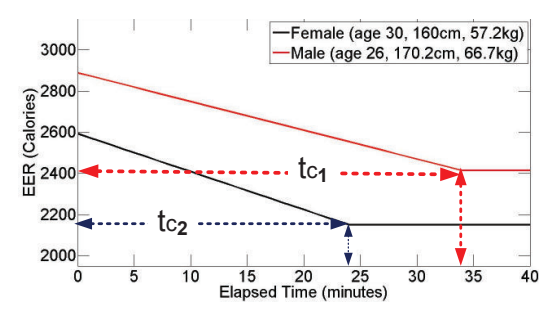

Figure 2: The EER example of two people the comfort index from our TCC model with the actual feedback of occupants.

Referring to the World Health Organization (WHO) [32], we classify the occupants into three categories according to their body mass index (BMI): i) underweight (UW), ii) normal (NL), and iii) overweight (OW). The formula of BMI is as follows, and the BMI index of each group is shown in Table 3 .

$$
B M I=\frac{\text { Weight }(\mathrm{kg})}{(\text { Height }(\mathrm{m}))^{2}}
$$

In our experiment, 3 of the occupants were UW, 8 were NL and 2 were OW. Each round of training was carried out in the time when occupants arrived the office from outside environment. In each round of training, we assigned a fixed setpoint temperature, and occupants were asked to submit their feedback at a 5-minute interval. We adjusted the setpoint (indoor) temperature from $20{ }^{\circ} \mathrm{C}$ to $25^{\circ} \mathrm{C}$, with $0.5^{\circ} \mathrm{C}$ increment in each training.

The key parameters of our model for training are $a_{1}, b_{1}$ and $t_{c}$ of each occupant. We use linear regression that obtained from the training period and we estimate the comfort index of each occupant from our TCC model. The comparisons between the TCC model and the actual feedback of occupants are shown in Fig. 4. The accuracy for OW is $78 \%, \mathrm{NL}$ is $75 \%$ and UW is $67 \%$. The maximum error of the comfort index is only 1 . The result indicates that our TCC model is capable to estimate the comfort index of the three occupants.

Here we explain our model in details. Noted that the heat loss is primarily determined by the clothes of an occupant, and in our model, this is determined by the outdoor temperature (which is translated into approximately the clothing of an occupant). The heat generation, however, depends on the metabolic rate of an individual and this differs across different occupants (one may recall our three training parameters $a_{1}, b_{1}$ and $t_{c}$ are all for heat generation). We look into EER in more details. Fig. 3a shows one of the trainings, where one occupant is selected from each BMI group: occupant A from OW, occupant B from NL and occupant C from UW. Their change of EER are shown in Fig. 3b. Fig. 3a and $3 \mathrm{~b}$ indicate that there is a strong correlation between the change of EER with the comfort index of occupants. More specifically, occupants A, B and C have diverging comfort indices at the beginning: $\mathrm{A}$ and $\mathrm{B}$ are warm and slightly warm respectively, and $\mathrm{C}$ is neutral. Their differences can be explained by the $E E R_{s}$ values, which are 3100,2350 and 2100. Noted that the thermal sensations of occupants change with time, e.g., occupant A becomes neutral after 30 minutes, whereas $\mathrm{B}$ and $\mathrm{C}$ are both slightly cool after 40 and 25 minutes respectively. It is worth noticing that the thermal sensations of $\mathrm{B}$ and $\mathrm{C}$ are close to each other as their $E E R_{e}$ are close to each other. The result verifies the connection between EER and comfort index of a person.

\section{THE SETPOINT OPTIMIZATION ALGO- RITHM}

The objective of the setpoint optimization algorithm (SOA) is to find out the optimal setpoint temperature to a group of occupants in a specific room, thus maximizing the overall thermal comfort of all occupants, where a certain percentage of occupants are staying within the comfort zone. Our 


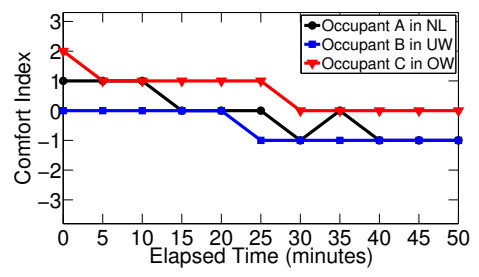

(a) Comfort index

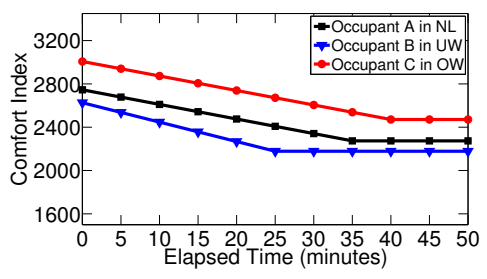

(b) EER

Figure 3: Connection between EER and Comfort index

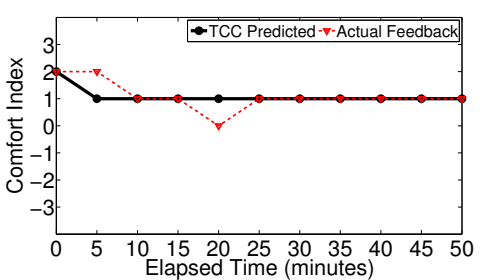

(a) OW group

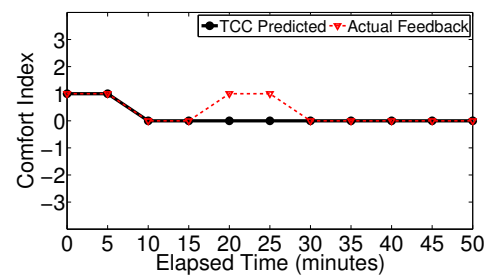

(b) NL group

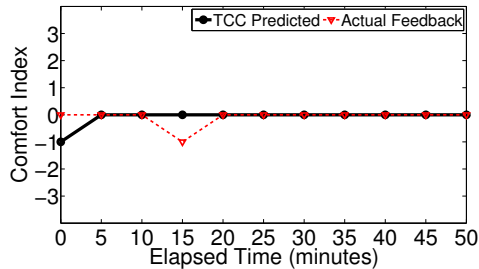

(c) UW group

Figure 4: TCC model validation under different BMI groups

algorithm is shown in Algorithm 1. There are three input parameters: $\mathbb{O}, r, T_{o}$. $\mathbb{O}$ is the set of occupants in a room; $r$ is the threshold percentage of occupants within the comfort zone (e.g., 80\%); and $T_{o}$ is the outdoor temperature. For every occupant $j \in \mathbb{O}$, there is a corresponding TCC model $C_{j}$ and a elapsed time $t_{j}$ after occupant $j$ enters the room.

Our algorithm first identifies the comfort temperature of each occupant. Then we compute the optimized setpoint temperature iteratively for all occupants. In each iteration, we determine a candidate of setpoint temperature and check whether this temperature satisfies the thermal comfort requirement of all occupants. If it fails, we adjust the set of occupants and proceed next iteration. In other words, we first calculate a candidate of setpoint temperature $T^{*}$ by minimizing the sum of comfort index of all occupants. Then we count the numbers of occupants who feel comfortable, denoted as $N$. If the target requirement (more than $r|\mathbb{O}|$ occupants are satisfied) is met, the optimized setpoint $T^{*}$ is found. Otherwise, we eliminate an occupant whose preferred temperature is the farthest from the candidate setpoint temperature since we try to satisfy as many occupants as possible.

\section{SIMULATION}

\subsection{Simulation Setup}

We evaluated our system in two different scales. Firstly, we simulated a classroom with different occupants' profiles. Secondly, we adopted the academic calendar from The Hong Kong Polytechnic University (denoted PolyU thereafter) to evaluate our system in a large scale. We compare our system with fixed setpoint strategy.

In our simulation, we created the occupant profiles based on the results from our experiment in model validation. We picked one occupant from each BMI category. The profile of an occupant is denoted as $\left[a_{1}, b_{1}, t_{c}\right]$. Thus, profiles from occupant in OW category, NL category and UW category

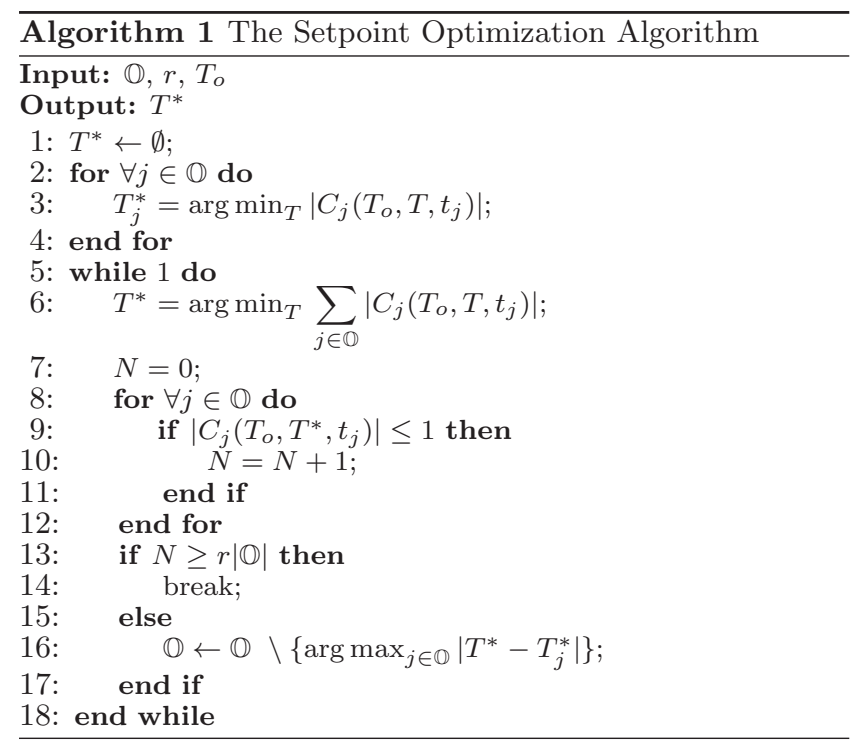

are $[0.0027,-4.99,30],[0.0041,-7.08,40]$ and $[0.002,-2.5325$, 25] respectively.

For the simulation in a classroom, we created two groups of occupants, named as group A and group B. Each group had 100 occupants. The ratio of OW:NL:UW in group A was 1:7:2, whereas group B was 1:1:3. The outdoor temperature was set to $30^{\circ} \mathrm{C}$. The class in each group lasted for an hour. The temperature under the fixed setpoint was $22^{\circ} \mathrm{C}$.

For the PolyU data, there were over 950 classes in every weekday. We evaluated our system with one whole year,and the classes were repeated every week. We adopted the outdoor temperature data of Hong Kong in 2013 from The Hong Kong Observatory [33] as the system input. From our field measurement, PolyU applied a fixed setpoint temperature of $22^{\circ} \mathrm{C}$ in summer period (May to October) and $24^{\circ} \mathrm{C}$ in winter period. 


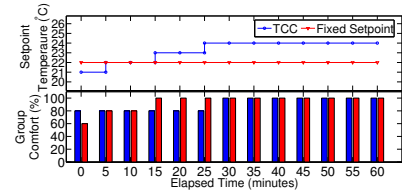

(a) Group comfort

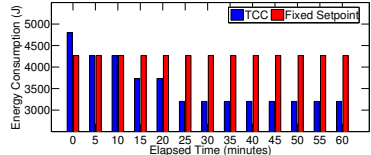

(b) Energy consumption
Figure 5: Group A simulation results

To compare the energy consumption, we adopted the energytemperature correlation model $P=\left|\frac{\lambda}{M}\left(T_{i}-T_{o}\right)\right|$ in [14]. We define $P$ as the energy consumed by HVAC system in onesecond, $\lambda$ as the conductivity of that particular classroom. Intuitively, the larger the $\lambda$, the less the heat preservation being trapped into the room. $M$ is energy transformation ratio of HVAC system, which is used to indicate the energy efficiency to the HVAC system. Again, $T_{i}$ is the actual room temperature, and $T_{o}$ is the average outdoor temperature. The model assumes that in a given room, $\frac{\lambda}{M}$ is fixed, and thus more energy is consumed when the room setpoint temperature is far away from the outdoor temperature. The values of $\lambda$ and $M$ for classrooms at PolyU are same as [14], where $M$ is 0.14 for all classrooms. There are 155 classrooms in PolyU main campus, and the details are summarized in Table 4. For the simulation in a classroom, we took the parameters of the classroom with a capacity of 100 seats.

Table 4: Classroom at PolyU

\begin{tabular}{cccc}
\hline Seats & No. & Size $(L \times W \times H, m)$ & $\lambda(J / s \cdot K)$ \\
\hline 20 & 8 & $4 \times 5 \times 3$ & 70.5 \\
40 & 42 & $8 \times 5 \times 3$ & 118.5 \\
60 & 67 & $6 \times 10 \times 3$ & 162 \\
80 & 10 & $8 \times 10 \times 3$ & 201 \\
100 & 4 & $10 \times 10 \times 3.3$ & 249 \\
150 & 17 & $10 \times 15 \times 4$ & 375 \\
200 & 5 & $15 \times 14 \times 5$ & 533 \\
300 & 2 & $15 \times 20 \times 6$ & 765 \\
\hline
\end{tabular}

\subsection{Simulation Results}

\subsubsection{Classroom simulation result}

Figure 5 shows the result of group A. With the starting setpoint calculated by $\mathrm{OPTC}$ at $21^{\circ} \mathrm{C}$, it progressively increases with time and higher than the fixed setpoint, which is $22{ }^{\circ} \mathrm{C}$ after 15 minutes. We can see that OPTC achieves group thermal comfort requirement $(\geq 80 \%)$ all the time, while fixed setpoint fails to meet the requirement in the first few minutes.

The energy consumption is shown in Fig. 6b. Since fixed setpoint does not changes its setpoint, its energy consumption is steady. For OPTC, when the setpoint is changed, the energy consumption drops dramatically. For the last 40 minutes, OPTC consumes only $35 \%$ of the energy of fixed setpoint. As a whole, there is a reduction of $16.5 \%$ energy consumption under OPTC.

The result of group B is shown in Fig. 6. Compared to that of group A, the group thermal comfort under fixed setpoint is far from satisfaction when the class begins. Only $20 \%$ of the students are within the comfort zone. In contrast, OPTC brings all the students stay within the comfort zone

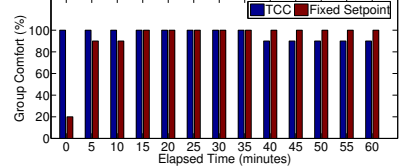

(a) Group comfort

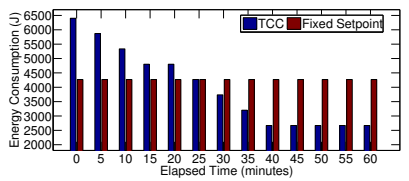

(b) Energy consumption
Figure 6: Group B simulation results

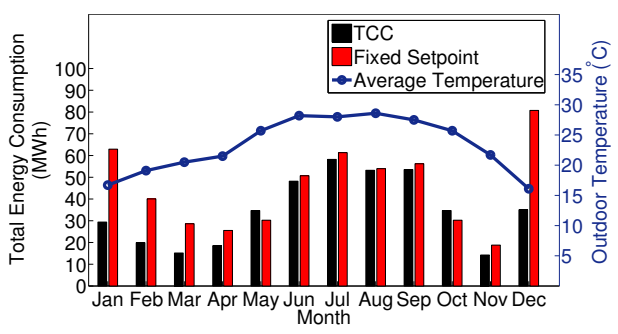

Figure 7: Energy consumption in one year

for more than $60 \%$ of the time, and it maintains $90 \%$ of group comfort for the students in class. From the results, beside the large differences of energy consumption between OPTC and fixed setpoint, it is obvious that group B saves even more energy. It can be explained by the ratio of UW is more than the OW in group B, which the setpoint temperatures from OPTC are generally higher than group A.

\subsubsection{Simulation of annual energy consumption}

The results of monthly energy consumption are shown in Fig. 7. The maximum and minimum averaged monthly temperature are $14^{\circ} \mathrm{C}$ and $31.1^{\circ} \mathrm{C}$ respectively. OPTC outperforms the fixed setpoint in 10 months with an exception in May and October. During summer period, the difference between OPTC and fixed setpoint are approximately $5 \%$. However, such differences enlarged rapidly when the outdoor temperature drops, especially at its bottom in January and December, the fixed setpoint consumes more than twice of the energy than OPTC. When compared with the fixed setpoint, OPTC saves $23.1 \%$ annual energy consumption.

\section{IMPLEMENTATION DETAILS}

We implemented a prototype of OPTC in buildings. The system workflow is shown in Fig. 8. The system is deployed in an OPTC server. The event monitor module in OPTC server collects data from the environment and occupants. After the optimal setpoint temperature is calculated, the building control module requests BMS to adjust the room temperature. To collect data from occupants, we developed a mobile application. The occupants provide their thermal feedback and base rooms to the OPTC server via the mobile application. Besides collecting data from the occupants, the OPTC server communicates with the BMS for two objectives: 1) to collect the indoor temperature and the outdoor temperature; 2) to control the indoor temperature of a room. We discuss each part in details as follows.

\subsection{A Mobile Application for Occupants}

The mobile application collects the following information: 


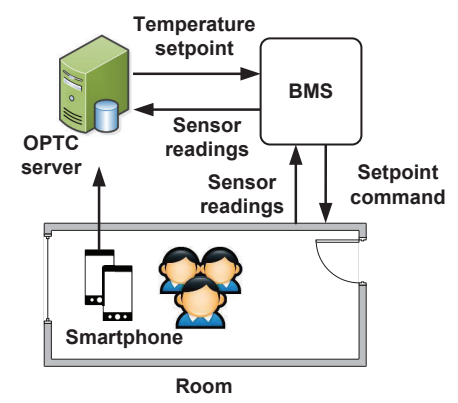

Figure 8: The system workflow of OPTC

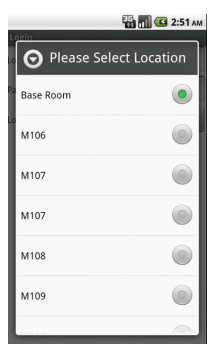

(a) Location selection

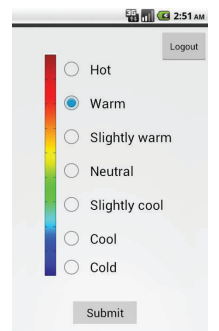

(b) Voting screen
Figure 9: The mobile application for occupants

1) Occupant identity information; as explained before, our system requests occupant registration. This registration is one-off and collects data such as weight, age, gender, etc of the occupants. Recalled from Section 4, this information are needed for our TCC model.

2) The occupants are required to register their base room$\mathrm{s}$ as shown in Fig. 9a; This registration is also one-off. If the occupant has only one base room, all the computation and adjustment will be based on this room. Noted that in this situation, the setpoint temperature still requires adjustments from time to time, and primarily non-intrusive. If the room hosts multiple occupants, the setpoint optimization algorithm needs to be applied. If an occupant has multiple base rooms, we need to obtain his location from time to time. To minimize the inputs from the occupant, the location can be obtained either from his meeting schedule, or a location detection algorithm can be applied. In a commercial office setting, an occupant usually has few numbers of base rooms. This makes the challenge for the location detection algorith$\mathrm{m}$ reasonable. The detailed location detection algorithm is out of the scope of our paper. In Section 8, our experiment is solely confined to the single room case.

3) Occupants thermal sensation (i.e., their votes); when our model-driven adjustment is not able to satisfy occupant's comfort, the occupant can provide feedback using the mobile application as shown in Fig. 9b, which follows the 7point thermal comfort index design as explained in Section 4.3 .

\subsection{Data Collection and Temperature Control in Building}

In a typical HVAC system, there are thousands of sensors to monitor the equipment status and condition feedback from the serving areas [11]. The temperature sensors are normally mounted on walls or at the ceiling of room. There are also sensors installed outside the buildings to col- lect outdoor sensors data. Both of these data are sent to BMS through a network. Our OPTC requires the indoor and outdoor temperature. Since these data are available in the existing BMS, we retrieve such data directly from the BMS [18].

Besides the data collection at BMS, we also need to control the setpoint temperature of rooms. This function is realized through the Building Automation and Control Networks (BACnet) protocol [34] in our OPTC framework [35]. Given that BACnet is the most dominant communication protocol in BMS today, we believe our system can be widely adopted into different buildings.

\section{EXPERIMENT}

\subsection{Experiment Setup}

We conducted experiments in our university and a A-class commercial office. In our university, the air-conditioning of lecture theaters were controlled by the BMS, and the lecture theatre in our experiment had a capacity of 130 people at the building of Y-core.

For the commercial office in our experiment, the provision of air-conditioning was $24 / 7$, the floor plan and the size of rooms are shown in Fig. 13. There were 5 individual rooms (room A to E) and 3 meeting rooms (room 1 to 3 ).

We set up our OPTC server on campus and connected both the BMSes of campus and office using virtual private network (VPN) since the campus network was located at Intranet. To collect the votes of students, we designed the mobile application as discussed in Sec. 7.1.

We evaluate our scheme through three performance metrics: 1) the improvement of thermal comfort of occupants; 2 ) the missing rate in satisfying group thermal comfort. We adopt a threshold of $80 \%$ following the ASHRAE standard; and 3) energy conservation.

\subsection{Experiment results in our university}

We conducted our experiment during a three-hour lecture in our university. 87 students were participated in the experiment, and the outdoor temperature was $31.4^{\circ} \mathrm{C}$. From our pre-measurement, the university has a fixed setpoint temperature of $21.5^{\circ} \mathrm{C}$. We divided the lecture into two one-hour sessions, where we experimented the fixed setpoint approach in the first session, and TCC approach in the second session.

Before the class, students were guided to install our smartphone application. They were instructed to provide feedback regarding their thermal sensations (as shown in Table 1) at an interval of 10 minutes. To develop the TCC model of each student, we also collected student's age, height and weight respectively.

Results of different group comfort under the fixed setpoint and our TCC modeled setpoint are shown in Fig. 10. The

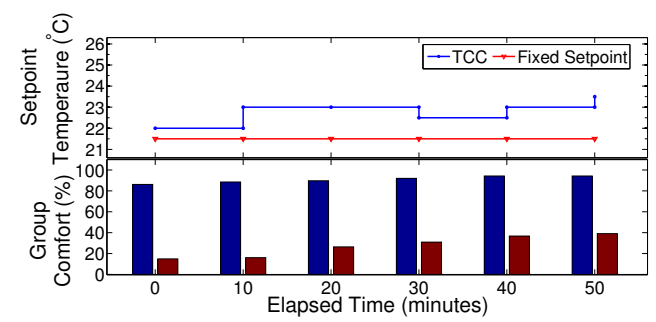

Figure 10: Setpoint temperature and group comfort 


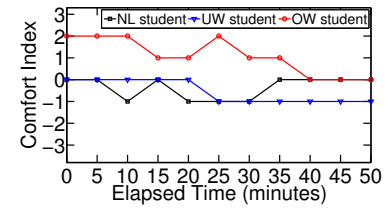

(a) Comfort index

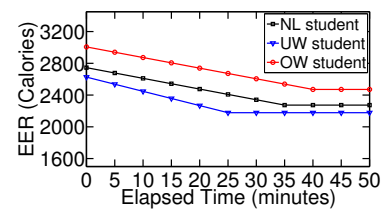

(b) EER
Figure 11: Students from different BMI groups

$\mathrm{x}$-axis is the elapsed time starting from the students arriving at the lecture theater. The y-axis of the upper part of the figure is the corresponding setpoint given by the fixed setpoint approach and our TCC approach; and the y-axis of the lower part of the figure is the overall feedback of students.

We compare the two setpoint approaches. In the experiment on fixed setpoint approach, more than $85 \%$ of the students were not at a comfort condition when they were just arrived the lecture theatre. In other words, their comfort indices were outside the comfort zone. 25 students even voted 3 , and only 5 students voted thermal neutrality. At the time of 40 minutes, fixed setpoint has achieved its highest group comfort, which is still only about $40 \%$ of the students.

We then applied our model by first creating their profile using the TCC model with the given data. We categorized the students into three groups according to their BMIs. We selected one student from each group and showed their comfort indices and EER in Fig. 11a and $11 \mathrm{~b}$ respectively. It was not surprised that students in OW group had a higher $E E R_{s}$, followed by the student in NL group, who had a relatively mild change of $E E R$ with time. The student in the UW group had both the highest and least $E E R_{s}$ and $E E R_{e}$ respectively among the other groups.

To compare the improvement brought by our TCC model, we started to adjust the setpoint temperature of the lecture theater via our OPTC server after the 10-minute break at our second-hour experiment session. Students were again told to vote at every 10 minutes. Again, in Fig. 10, when the elapsed time was between 0 and 10 minutes, the TCC setpoint $\left(22^{\circ} \mathrm{C}\right)$ was slightly higher than the fixed setpoint, and the difference enlarged between 10 to 30 minutes. The result showed that there was a great improvement to their conditions of thermal comfort as compared with the default fixed setpoint at $21.5^{\circ} \mathrm{C}$. Only 9 students (10.3\%) were not at the comfort zone in the first 20 minutes, and later reduced to 5 students $(5.7 \%)$ after 30 minutes. Our TCC approach was able to achieve $80 \%$ group comfort throughout the whole period. As compared with the group comfort before the TC$\mathrm{C}$ model was applied, there was an average of $63 \%$ thermal comfort improvement to the students in our experiment.

\subsection{Experiment results in a commercial office}

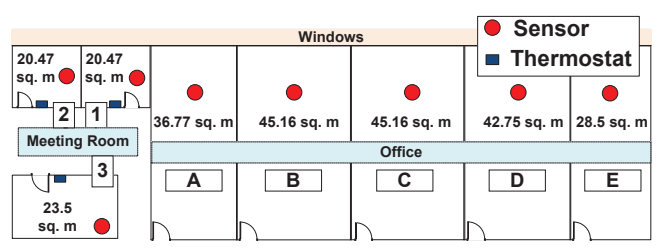

Figure 13: Floor plan of office

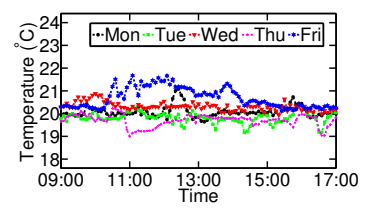

(a) Room B

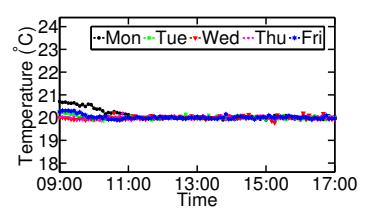

(b) Room E
Figure 12: Temperature of two office rooms

In this part, we discuss the experiment conducted in a commercial office. To study the existing indoor temperature and occupants comfort, we initially deployed three temperature sensors at different rooms for three weeks aiming to study the trend of temperature change under different outdoor temperatures. We then carried out two five-day sessions, one for the fixed setpoint approach and one for our TCC approach.

Before going into the analysis, we first present several interesting observations. Firstly, rooms with more people (e.g., room B with 13 people), experienced more significant temperature changes than rooms with less people (e.g., room E with 2 people). Fig. $12 \mathrm{a}$ and $12 \mathrm{~b}$ illustrate this phenomenon in room $\mathrm{B}$ and $\mathrm{E}$ respectively from one sample day during working hours.

Secondly, we observed that the temperature differences in a room can be as much as $2.5^{\circ} \mathrm{C}$; such differences may contribute to occupant discomfort. We traced the reasons and found that areas with printer and computers were the main culprits for a warmer temperature; and during noon time, the areas near windows were affected by the sunlight and thus created a small warm zone.

These findings show that the placement of temperature sensors have a direct effect to the control accuracy of BM$\mathrm{S}$ and thus the room temperature. The number of sensors and location should be carefully considered; otherwise, occupants will be modeled with bias.

Surprisingly, we also observed that it took approximately 4.5 minutes in average for a room to reach a setpoint temperature. From our discussion with building services engineers, it takes time for the setpoint adjustment since the chilled water and the air flow from the air-conditioning terminal units (e.g., fan coil unit and variable air volume box) need time to work together to attain the desired setpoint. This time-lag varies with building designs and air-conditioning systems. As such, in our experiment, the setpoint temperature was determined by the TCC model at every 5 -minute.

Room B was chosen in our experiment as it has the highest and most steady occupancy. Two smart sensors TelosB were additionally deployed in room to provide a finer temperature measurement. The default setpoint temperature was fixed at $22^{\circ} \mathrm{C}$ (summer period) by the facility management of the building. We carried out two 5-day (Monday to Friday) measurements. We studied the fixed setpoint approach in the first measurement. Occupants were invited to provide feedback using our smartphone application anytime when they felt there was an obvious change to the thermal sensation.

We finally collected a total of 403 votes, which were fairly distributed from the 13 occupants. The results are shown in Fig. 14a. The central red line is the median, and the height of the box is the inter-quartile range of the votes, where the top and bottom of the boxes are the $75^{\text {th }}$ and $25^{\text {th }}$ percentile 


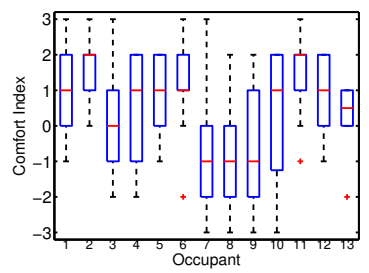

(a) Before using OPTC

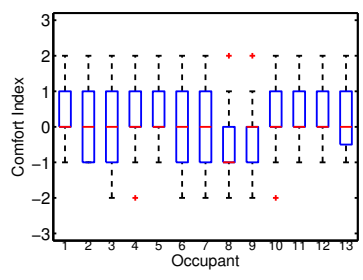

(b) After using OPTC
Figure 14: Feedback from office experiment

of the votes. Extreme data that are considered outliers is shown using the "whiskers", i.e., with a "plus" sign.

There were dissatisfactions from the occupants to their existing fixed setpoint temperature. Around $45 \%$ of the votes were outside the comfort zone, and 7 votes (7.4\%) were even at the extreme comfort index ( -3 or 3$)$.

We then deployed our OPTC for comparison. With the previous feedback and information of occupants (i.e., age, weight and height), we built the TCC model for each occupant. We conducted another 5-day experiment, and collected 334 votes.

The results are shown in Fig.14b. There was a major improvement of the occupant thermal comfort. $89 \%$ of the votes were within the comfort zone and no votes was in the extreme range ( -3 or 3$)$. There were 115 votes with thermal neutrality and they were fairly distributed from the 13 occupants. 12 occupants had a median of comfort neutrality, compared with 1 in our first week experiment. The overall improvement was $33.8 \%$.

We further display one of the experiment days in the timeline format as shown in Fig. 15. There are two parts in the figure. The upper part shows the setpoint adjustment under fixed setpoint approach and our TCC approach. The lower part shows the group thermal comfort from the votes of occupants. The average outdoor temperature was $30.52^{\circ} \mathrm{C}$ during the day of experiment, with a diurnal difference of $3.17^{\circ} \mathrm{C}$. Obviously, our TCC model has maintained a higher level of thermal comfort to the occupants than the fixed setpoint as shown in the group comfort percentage. Noted that the fixed setpoint failed to meet the target of group comfort during that experiment (i.e., $80 \%$ ), whereas our TCC model was able to achieve $70 \%$ of the time meeting the requirement.

Beside the improvement of thermal comfort, there is also better energy performance. With the baseline of setpoint temperature at $22^{\circ} \mathrm{C}$, there was an average of $1.75^{\circ} \mathrm{C}$ setpoint increment during the experiment period. Studies indicate that one-degree setpoint difference yields around $10 \%$ difference on energy use [36]. More specifically, we consider the energy input for the air-conditioning terminal units $(\mathrm{kWh})$,

$$
\sum_{i=1}^{n}\left\{\left(\frac{\dot{m}_{i} c \Delta T_{i}}{\eta_{i} \cdot C O P}+P_{f_{i}}\right) h r_{i}\right\},
$$

where $\dot{m}$ is the air mass flow rate $(\mathrm{kg} / \mathrm{s})$, c is the specific heat capacity of air $(\mathrm{kJ} / \mathrm{kgK}), \Delta \mathrm{T}$ is the difference between supply and return air temperature $(\mathrm{K}), \eta$ is the heat transfer efficiency of the air-conditioning unit using chilled water, $P_{f}$ is the operating fan motor power, $\mathrm{COP}$ is the coefficient of

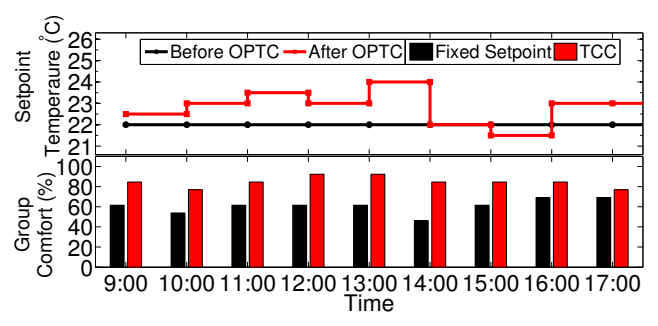

Figure 15: Setpoint and group comfort in office

performance of the central chiller plant and $h r$ is the cooling duration (hours).

We calculate the energy input by using the operating logs of BMS every 5-minute interval (i.e., $h r=1 / 12$ ). By assuming that the operating conditions were the same during the experiment, we can derive that our OPTC scheme was able to save $18 \%$ of energy consumption of the air-conditioning terminal units.

\section{CONCLUSION}

In this paper, we present an occupant-participatory thermal comfort framework. This framework incorporates occupants feedback into the loop of air-conditioning adjustment decisions. In the core of this framework, we developed a temperature-comfort correlation (TCC) model, capturing users favorite temperatures non-intrusively from their daily environment. Our model adopts the spirits of traditional PMV index and the adaptive approach developed from built environment. Nevertheless, to make sure that the model can fit in daily usage, we make certain modifications where the model input data can be easily collected so that the occupant has incentives to participate. We also developed an algorithm which resolves diverging comfort requirements of multiple people. We have a full set of field validations, comprehensive simulations and real world experiments. The results showed that we can maintain thermal comfort while reducing energy consumption for $18 \%$.

\section{Acknowledgements}

This work is supported in part by National Natural Science Foundation of China (No. 61272464), RGC/GRF PolyU 5264/13E, HK PolyU G-YM06, A-PK95, 1-ZVC2.

\section{REFERENCES}

[1] Electrical and Mechanical Service Department (EMSD), Hong Kong, http://www.emsd.gov.hk/emsd/eng/pee/edata_1.shtml.

[2] A. Aswani, N. Master, J. Taneja, D. Culler, and C. Tomlin. Reducing transient and steady state electricity consumption in hvac using learning-based model predictive control. 100(1):240-253, 2012.

[3] Carl Ellis, James Scott, Mike Hazas, and John C. Krumm. Earlyoff: Using house cooling rates to save energy. In Proc. ACM BuildSys '12.

[4] Y. Agarwal, B. Balaji, S. Dutta, R. Gupta, and T. Weng. Duty-cycling buildings aggressively: The next frontier in hvac control. In Proc. ACM/IEEE IPSN'11.

[5] Y. Agarwal, B. Balaji, R. Gupta, J. Lyles, M. Wei, and T. Weng. Occupancy-driven energy management for smart building automation. In Proc. ACM BuildSys '10. 
[6] Varick L. Erickson and Alberto E. Cerpa. Thermovote: participatory sensing for efficient building hvac conditioning. In Proc. ACM BuildSys '12.

[7] Xinlei Wang, Wei Cheng, P. Mohapatra, and T. Abdelzaher. Artsense: Anonymous reputation and trust in participatory sensing. In Proc IEEE INFOCOM 13'.

[8] Delphine Christin, Andreas Reinhardt, Salil S. Kanhere, and Matthias Hollick. A survey on privacy in mobile participatory sensing applications. Journal of Systems and Software, 84(11):1928 - 1946, 2011.

[9] Xiaofan Jiang, Stephen Dawson-Haggerty, Prabal Dutta, and David Culler. Design and implementation of a high-fidelity AC metering network. In Proc. ACM IPSN '09.

[10] Stephen Dawson-Haggerty, Steven Lanzisera, Jay Taneja, Richard Brown, and David Culler. @scale: insights from a large, long-lived appliance energy WSN. In Proc. ACM IPSN '12.

[11] Stephen Dawson-Haggerty, Andrew Krioukov, Jay Taneja, Sagar Karandikar, Gabe Fierro, Nikita Kitaev, and David Culler. BOSS: building operating system services. In Proc. USENIX NSDI'13.

[12] Marija Milenkovic and Oliver Amft. An opportunistic activity-sensing approach to save energy in office buildings. In Proc. ACM e-Energy '13.

[13] Jiakang Lu, Tamim Sookoor, Vijay Srinivasan, Ge Gao, Brian Holben, John Stankovic, Eric Field, and Kamin Whitehouse. The smart thermostat: using occupancy sensors to save energy in homes. In Proc. ACM SenSys '10.

[14] Yi Yuan, Dawei Pan, Dan Wang, Xiaohua Xu, Yu Peng, Xiyuan Peng, and Peng-Jun Wan. A study towards applying thermal inertia for energy conservation in rooms. ACM Trans. Sen. Netw., 10(1):7:1-7:25, December 2013.

[15] Nol Djongyang, Ren Tchinda, and Donatien Njomo. Thermal comfort: A review paper. Renewable and Sustainable Energy Reviews, 14(9):2626 - 2640, 2010.

[16] Fanger PO. Thermal comfort, analysis and application in environmental engineering. Copenhagen: Danish Technical Press, 1970.

[17] de Dear, G. Brager, and D. Cooper. Developing an adaptive model of thermal comfort and preference. ASHRAE Trans., V.104(1a)(1a):145-167, 1998.

[18] Hang-Yat Lam Abraham and Dan Wang. Carrying my environment with me: A participatory-sensing approach to enhance thermal comfort. In Proc. ACM BuildSys '13.

[19] Peter Xiang Gao and S. Keshav. Optimal personal comfort management using spot+. In Proc. ACM BuildSys '13.

[20] Farrokh Jazizadeh and Burcin Becerik-Gerber. Toward adaptive comfort management in office buildings using participatory sensing for end user driven control. In Proc. ACM BuildSys '12.

[21] A.P. Gagge, J.A.J. Stolwijk, and B. Saltin. Comfort and thermal sensations and associated physiological responses during exercise at various ambient temperatures. Environmental Research, 2(3):209 - 229, 1969.

[22] T. Goto, J. Toftum, R. Dear, and P.O. Fanger. Thermal sensation and thermophysiological responses to metabolic step-changes. International Journal of Biometeorology, 50(5):323-332, 2006.

[23] J. F. Nicol and M. A. Humphreys. New standards for comfort and energy use in buildings. Building Research 85 Information, 37(1):68-73, 2009.

[24] A.P. Gagge, A.P. Fobelets, and L.G. Berglund. A standard predictive index of human response to the thermal environment. Jan 1986.

[25] ASHRAE standard 55-2010:Thermal Environmental Conditions for Human Occupancy. ASHRAE, 2010.

[26] Innova Air Tech Instruments. Thermal Comfort. Innova Air Tech Instruments, 2002.
[27] Craig Morgan and Richard de Dear. Weather, clothing and thermal adaptation to indoor climate. Climate Research, 24(3):267-284, 2003.

[28] K Krauchi and ANNA Wirz-Justice. Circadian rhythm of heat production, heart rate, and skin and core temperature under unmasking conditions in men. American Journal of Physiology-Regulatory, Integrative and Comparative Physiology, 267(3):R819-R829, 1994.

[29] John R Speakman. Body size, energy metabolism and lifespan. Journal of Experimental Biology, 208(9):1717-1730, 2005.

[30] Institute of Medicine. Dietary Reference Intakes for Energy, Carbohydrate, Fiber, Fat, Fatty Acids, Cholesterol, Protein, and Amino Acids (Macronutrients). The National Academies Press, 2005.

[31] G.S. Brager and R.J. de Dear. Climate, comfort \& natural ventilation: A new adaptive comfort standard for ashrae standard 55. In Proc. Moving Thermal Comfort Standards into the 21st Century 01'.

[32] World Health Organization. www.who.int/bmi/, 2014.

[33] Hong Kong Observatory. www.hko.gov.hk/contente.htm, 2014.

[34] ASHRAE standard 135-2010:A Data Communication Protocol for Building Automation and Control Networks. ASHRAE, 2010.

[35] Qinghua Luo, A.H.-Y. Lam, Dan Wang, D.W.-T. Chan, Yu Peng, and Xiyuan Peng. Demo abstract: Towards a wireless building management system with minimum change to the building protocols. In Proc. ICCPS '12.

[36] Office of Environment and Heritage (OEH), NSW, www.ehp.qld.gov.au/sustainability/sector-guides/energyuse.html.

\section{Appendix}

We discuss the approach to compute $a_{1}$ and $b_{1}$ in Section 4.2.1. We use linear regression to process data in period $t \in\left[0, t_{c}\right]$. Combined with Eq. 4, Eq. 1 can be written as:

$$
C\left(T_{i}, T_{o}, t\right)=a_{1} \times E E R(t)+b_{1}+L\left(T_{i}, T_{o}\right)
$$

With Eq. 3, we have:

$$
C\left(T_{i}, T_{o}, t\right)=\hat{a} \times t+\hat{b}+L\left(T_{i}, T_{o}\right) \quad t \in\left[0, t_{c}\right]
$$

where $\hat{a}=-a_{1}\left(E E R_{s}-E E R_{e}\right) / t_{c}$ and $\hat{b}=a_{1} \times E E R_{s}+b_{1}$.

From Eq. $5, L\left(T_{i}, T_{o}\right)$ is computed given the values of $T_{i}$ and $T_{o}$. Thus $C\left(T_{i}, T_{o}, t\right)-L\left(T_{i}, T_{o}\right)$ is linear to $t$ because the unknown parameters $(\hat{a}, \hat{b})$ in Eq. 8 are constants. When an occupant votes, we record $T_{i}, T_{o}, t$ and the voted thermal comfort index $v$. Ideally, the predicted index from our model equals to the voted index from occupant, which means that we expect $C\left(T_{i}, T_{o}, t\right)=v$. We can then use the linear regression technique with collected data $\left\langle T_{i}, T_{o}, t, v\right\rangle$ to obtain $\hat{a}$ and $\hat{b}$. Finally, $a_{1}$ and $b_{1}$ can be computed from $\hat{a}$ and $\hat{b}$ since $E E R_{s}, E E R_{e}$ and $t_{c}$ are known. 